\title{
Complejo de Shone
}

\section{René Oswaldo Villaseñor Díaz, ${ }^{1}$ Alfonso García Luna, ${ }^{2}$ María Campos Escoto, ${ }^{3}$ Rosa Ponce Partida, ${ }^{3}$ Luis Gerardo Domínguez Carrillo ${ }^{4}$}

\section{Resumen}

Antecedentes: El denominado complejo de Shone es un conjunto de anormalidades congénitas cardiacas que involucran el lado izquierdo del corazón, muy poco frecuente y de diagnóstico extraordinario en adulto. Caso Clínico: Femenino de 21 años que acudió a urgencias con disnea aguda. Se llegó al diagnóstico de complejo de Shone por ecocardiografía, estando presentes cinco niveles de obstrucción del corazón izquierdo que incluían: obstrucción supramitral, estenosis mitral, estenosis subaórtica, estenosis valvular aórtica y coartación aórtica. Se agregaron trombos en aurícula y ventrículo izquierdos; el cuadro mostró asociación a síndrome de preexcitación, la evolución llevó a su deceso en un lapso de cuatro horas a pesar de manejo intensivo.

Palabras clave: Complejo de Shone.

\section{INTRODUCCIÓN}

El denominado complejo de Shone ${ }^{1}$ (CS) descrito en 1963 es un conjunto de anormalidades congénitas cardiacas, cuando está completo se caracteriza ${ }^{2}$ por: a) presencia de membra-

${ }^{1}$ Cardiólogo, Unidad de Cuidados Intensivos del Hospital Ángeles León.

2 Jefe de la Unidad de Cuidados Intensivos del Hospital Ángeles León.

3 Residente de Imagenología, Departamento de Imagenología del Hospital Ángeles León.

${ }^{4}$ Especialista en Medicina de Rehabilitación. Hospital Ángeles León.

Correspondencia:

Dr. René Oswaldo Villaseñor Díaz

Correo electrónico: dragulea7@hotmail.com

Aceptado: 31-08-2016.

Este artículo puede ser consultado en versión completa en http:// www.medigraphic.com/actamedica

\section{Summary}

Background: Shone complex is a set of congenital cardiac abnormalities involving the left side of the heart, it is infrequent, and extraordinary in adult life. Clinical case: Female 21 who visited the emergency department with acute dyspnea, in whom the diagnosis was Shone complex by echocardiography, finding five levels of obstruction of the left heart, including: supramitral obstruction, mitral stenosis, subaortic stenosis, aortic stenosis and aortic coarctation; finding also like complication thrombi in left atrium and ventricle, associated with preexcitation syndrome; her evolution was torpid with death over a period of four hours despite intensive management.

Key words: Shone complex.

na sobre la válvula mitral del corazón formando un anillo supravalvular que impide el correcto flujo sanguíneo desde la aurícula izquierda al ventrículo izquierdo; b) válvula mitral "en paracaídas" debido a su aspecto, ya que la válvula se abre hacia un solo músculo papilar; c) obstrucción subaórtica y d) coartación aórtica. Estas anomalías afectan fisiológicamente la entrada y salida del flujo sanguíneo del ventrículo izquierdo, ${ }^{3}$ originando incremento de la presión en las cámaras cardiacas izquierdas y su magnitud está relacionada con el grado de severidad. El complejo de Shone puede aparecer de manera incompleta, existiendo obstrucción en la válvula mitral asociada a obstrucción subaórtica o a coartación aórtica, independientemente la característica principal requerida para el diagnóstico es la existencia de lesión obstructiva en la válvula mitral, ${ }^{4}$ la cual puede manifestarse como anillo supramitral, mitral en paracaídas, con un solo músculo papilar, estenosis mitral congénita típica o estenosis mitral hipoplásica.

\section{CASO CLÍNICO}

Paciente femenina de 21 años, de medio socioeconómico medio-bajo, con antecedentes de dificultad para subir de peso desde la infancia y cuadros de vías respiratorias muy 
frecuentes (al menos cinco a seis por año) caracterizadas por tos no productiva y fatiga, relata que durante sus años escolares no podía efectuar ejercicios ni deporte al igual que sus compañeros por disnea. Menarca a los 16 años, con ritmo irregular y núbil, al interrogatorio dirigido mencionó disnea de medianos esfuerzos de seis meses de evolución, consultó a facultativo quien diagnosticó estenosis mitral de origen reumático, le prescribió diurético mostrando mejoría. Acudió a urgencias por sufrir de inicio súbito: disnea a pequeños esfuerzos, diaforesis y sensación de palpitaciones. A la exploración: paciente angustiada, peso 39 kilogramos, talla $150 \mathrm{~cm}$, IMC de 17.3 y en área torácica el ápex se encontró en el sexto espacio intercostal izquierdo sobre la línea axilar anterior. A la auscultación: soplo expulsivo aórtico grado IV/IV trasmitido a ambas carótidas; el primer ruido bajo en tono con soplo mesodiastólico grado III/IV. Abdomen
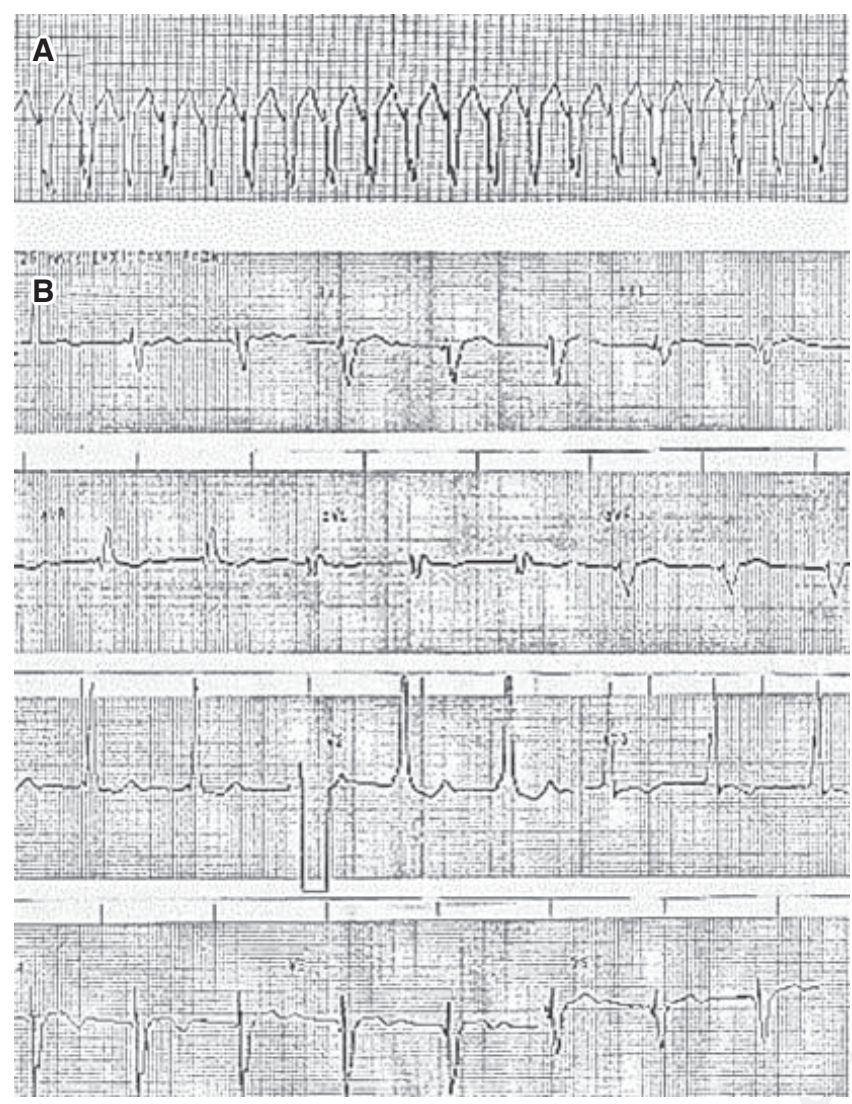

Figura 1. Electrocardiograma que muestra en A. Taquicardia supraventricular de 240 Ipm y vía accesoria oculta sugestiva de localización posteroseptal izquierda con conducción retrógrada. En B. Posterior a amiodarona y cardioversión eléctrica obteniendo: ritmo sinusal con frecuencia cardiaca 66 Ipm, AQRS en cuadrante superior derecho, bloqueo de rama derecha, PR menor de 0.12 seg. y presencia de ondas delta. normal, edema discreto en extremidades por debajo de rodilla con Godet +; no se detectaron pulsos arteriales en extremidades pélvicas estando presentes en las torácicas con $140 \mathrm{lpm}$. La tensión arterial en extremidad torácica superior derecha en 90/60 y los campos pulmonares con estertores crepitantes generalizados. Oximetría de pulso en mano derecha en $85 \%$ con aire ambiental que mejora a 94 con puntas de oxígeno a $3 \mathrm{~L} / \mathrm{min}$. Con impresión diagnóstica de coartación aórtica y lesión de válvula mitral se efectuó electrocardiograma, el cual reveló: taquicardia supraventricular de $240 \mathrm{lpm}$ y vía accesoria oculta sugestiva de localización posteroseptal izquierda con conducción retrógrada (Figura 1A). Se administró amiodarona y cardioversión eléctrica, obteniendo ritmo sinusal con frecuencia cardiaca $66 \mathrm{lpm}$, AQRS en cuadrante superior derecho, QRS ensanchado por bloqueo de rama derecha, PR menor de 0.12 seg. y presencia de ondas delta (Figura 1B).

El ecocardiograma reveló anillo supravalvular mitral semejante a cor triatriatum, pero sin condicionar obstrucción, sobre el cual se observó masa sugestiva de trombo de $35 \times 22 \mathrm{~mm}$.

Se halló trombo en aurícula izquierda que se debe a la velocidad lenta del flujo sanguíneo auricular por la obstrucción que condiciona la membrana supravalvular mitral (Figura 2A), además de imagen sugestiva de trombo de 24 x $18 \mathrm{~mm}$ en ápex de ventrículo izquierdo provocado por acinesia de los segmentos apicales del ventrículo izquierdo, condicionando estasis sanguínea y presencia de arritmia por el síndrome de preexcitación, con la consecuente

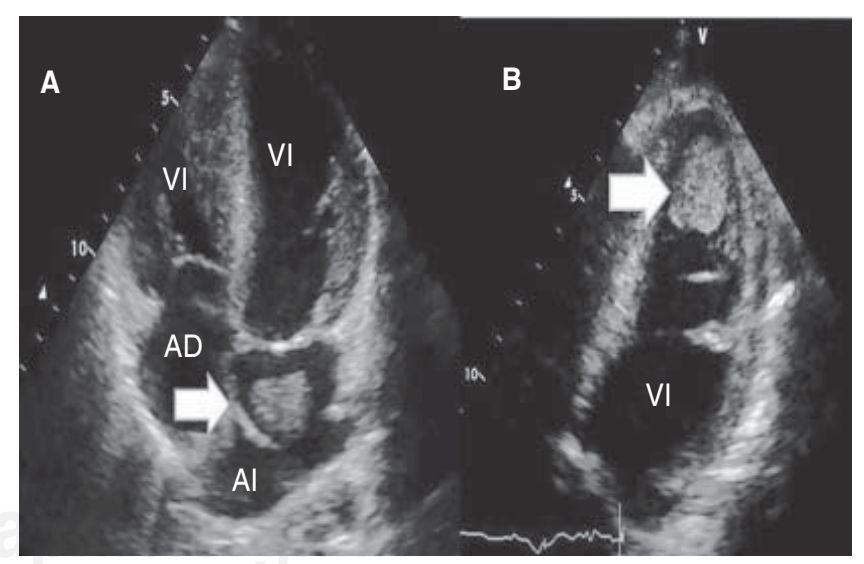

$A D=$ aurícula derecha; $A I=$ aurícula izquierda; $V D=$ ventrículo derecho; $\mathrm{VI}$ = ventrículo izquierdo.

Figura 2. Ecocardiograma en el que se aprecia en A. Anillo supravalvular mitral y trombo $35 \times 22 \mathrm{~mm}$ (flecha) intraauricular. En B. Imagen de trombo de 24 x $18 \mathrm{~mm}$ en ápex de ventrículo izquierdo (flecha). 


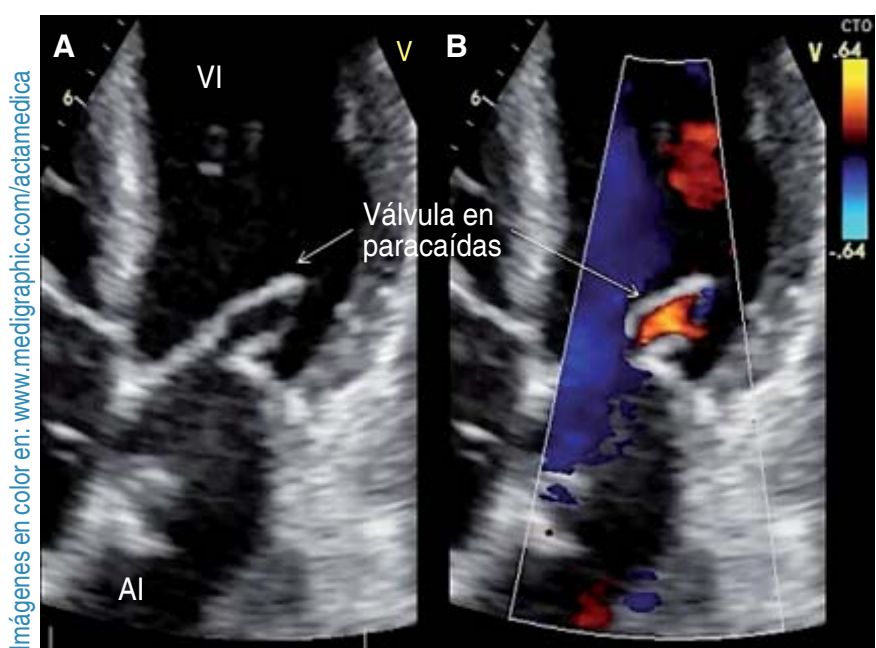

Figura 3. En A. Ecocardiograma en 2D, ventana apical de cuatro cámaras, en diástole, con válvula mitral en paracaídas, se observan cuerdas tendinosas que emergen de músculo papilar anterolateral. En B. Imagen comparativa con Doppler color, con área valvular de $1.2 \mathrm{~cm}^{2}$; tiempo de hemipresión de $185 \mathrm{~ms}$.

formación del trombo ventricular (Figura 2B). La válvula mitral estenótica con área valvular de $1.2 \mathrm{~cm}^{2}$; tiempo de hemipresión de 185 ms y con morfología en paracaídas (Figuras $3 A$ y $3 B$ ). Se detectó obstrucción subaórtica que condiciona estrechamiento del tracto de salida del ventrículo izquierdo y válvula aórtica bivalva con estenosis severa; con gradiente medio de $40 \mathrm{mmHg}$ y velocidad máxima de $4 \mathrm{~m} / \mathrm{seg}$; presión sistólica de la arteria pulmonar de $70 \mathrm{mmHg}$; además de hipoplasia del anillo aórtico y arco aórtico hipoplásico con coartación aórtica postductal y persistencia de conducto arterioso estimado en $21 \mathrm{~mm}$, corroborado por tomografía (Figuras $4 A$ y $4 B$ ). En un lapso de cuatro horas la paciente mostró insuficiencia cardiaca refractaria con edema agudo de pulmón, además de múltiples episodios de taquicardia supraventricular y posible desprendimiento de los trombos, ocasionando su deceso.

\section{DISCUSIÓN}

El caso que nos ocupa se trata de una cardiopatía compleja (desde el punto de vista embriológico) que no fue atendida en la infancia y permitió a la paciente llegar a la edad adulta. Pese a las manifestaciones clínicas desde entonces no fue diagnosticada hasta el momento de acudir a urgencias $y$, por su rápido deceso, no hubo la oportunidad de realizar otros estudios complementarios como ecocardiograma transesofágico y angiotomografía de corazón.

Al respecto, la estenosis en serie del corazón izquierdo se conoce como síndrome de Shone o complejo de Sho-

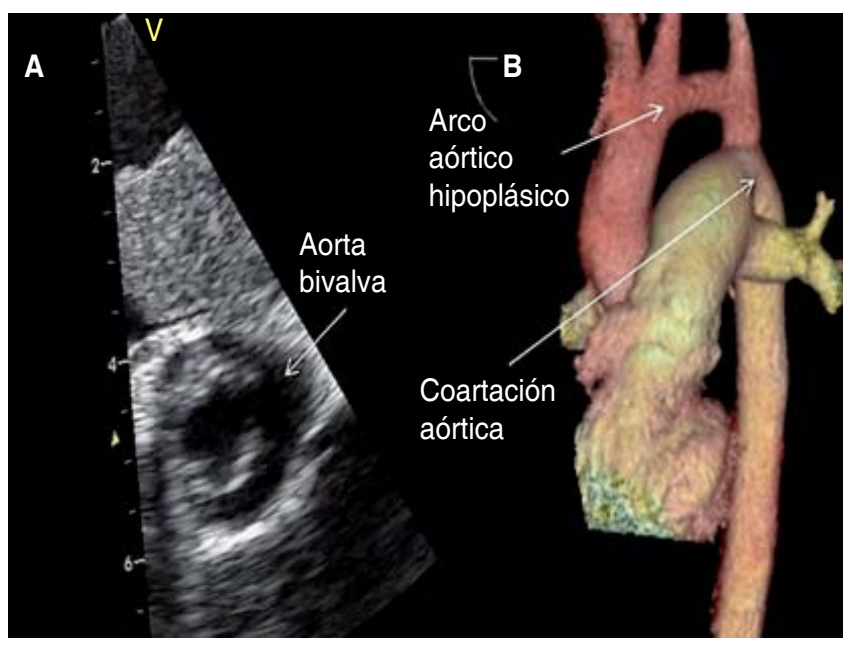

Figura 4. En A. Imagen ecocardiográfica que muestra aorta bivalva. En B. Reconstrucción tridimensional de aorta; cayado aórtico hipoplásico, coartación aórtica y persistencia de conducto arterioso, el cual ocasiona tracción a nivel ductal.

ne (CS), una entidad descrita en 1963 caracterizada por la presencia de cuatro anomalías congénitas: a) estenosis supravalvular mitral; b) válvula mitral en paracaídas; c) estenosis subvalvular aórtica y d) coartación aórtica. El CS puede aparecer de manera incompleta, con la existencia de obstrucción en la válvula mitral asociada a obstrucción subaórtica o a coartación aórtica, independientemente la característica principal que se requiere para el diagnóstico es la existencia de lesión obstructiva en la válvula mitral, la cual puede manifestarse como anillo supramitral, válvula mitral en paracaídas con un solo músculo papilar, estenosis mitral congénita típica o estenosis mitral hipoplásica. En la práctica clínica dentro de la definición se incluyen además afecciones estenóticas de las válvulas mitral y aórtica concomitantes con coartación aórtica. ${ }^{5}$ Estas anomalías afectan fisiológicamente la entrada y salida del flujo sanguíneo del ventrículo izquierdo, originando incremento de la presión en las cámaras cardiacas izquierdas. Su magnitud está relacionada con el grado de severidad. De las cuatro anormalidades presentes en el CS, la membrana mitral supravalvular es la primera en ocurrir, siendo el punto gatillo de desarrollo del resto de las anormalidades. ${ }^{6}$ El término de válvula mitral en "paracaídas"7 se debe a la apariencia de la válvula cuyas cuerdas cortas y gruesas se insertan en un solo músculo papilar, ${ }^{8}$ lo que implica restricción del movimiento valvular y obstrucción del flujo hacia el ventrículo izquierdo. El anillo supramitral está formado de tejido conectivo localizado por arriba de la base de las válvulas de la mitral que puede llegar a protruir en el orificio, provocando obstrucción fija. Por otra parte, el anillo supravalvular mitral está asociado a otros defectos en aproximadamente $90 \%$ de los casos. La 
estenosis subaórtica llega a observarse tanto de forma membranosa (22\%) como muscular (37\%), en ambos casos se presenta obstrucción de grado variable en la válvula aórtica, ocasionando obstrucción del flujo sanguíneo del ventrículo izquierdo hacia la aorta. Por último, puede apreciarse que la coartación aórtica incrementa la obstrucción del flujo del ventrículo izquierdo, esta última anomalía se asocia con mucha frecuencia a la válvula aórtica bivalva $(89 \%$ en la serie de Brown ${ }^{9}$ ), además la coartación de la aorta por sí sola se asocia a una anomalía de la válvula mitral entre 20 y $59 \%$ de los casos.

La etiología del CS se desconoce, la primera anomalía en aparecer es la obstrucción de la válvula mitral que surge desde la embriogénesis y provoca hipoplasia del ventrículo izquierdo con diferentes tipos de estenosis al flujo de salida del mismo. Con respecto a la epidemiología el CS es muy poco frecuente; ${ }^{10}$ la incidencia de cardiopatías congénitas en la población general es de aproximadamente 1\%; la incidencia del CS dentro de todas las malformaciones congénitas del corazón también es de alrededor de 1\%, por lo que nacen menos de 1 por cada 10,000 recién nacidos con esta patología.

Los síntomas aparecen durante los dos primeros años de vida y consisten en escasa ganancia de peso (no llega suficiente sangre oxigenada para alimentar los tejidos), taquipnea (se acumula líquido en los pulmones), cansancio y sudoración con las tomas. Los niños mayores presentan intolerancia a los esfuerzos físicos (como se relata en la historia clínica del caso), dado que la sangre se acumula en los pulmones y se bombea menos sangre a los músculos e hipertensión pulmonar. En algunos niños aparecen trastornos del ritmo cardiaco como consecuencia del crecimiento de la aurícula izquierda. Tanto en la niñez como en la adolescencia se incluye: falta de apetito (motivo del bajo peso y talla de la paciente que nos ocupa), tos (principalmente nocturna) infecciones respiratorias frecuentes, palidez (como resultado de anemia) fatiga, retención de líquidos, edema de extremidades inferiores, soplo cardiaco, disnea, diversos grados de cardiomegalia, hipertensión pulmonar e insuficiencia cardiaca congestiva. La severidad de los síntomas depende de la gravedad de la obstrucción del flujo sanguíneo y como característica importante los síntomas empeoran con el tiempo.

Las herramientas diagnósticas son el ecocardiograma ${ }^{11,12}$ que permite una valoración anatomofuncional del corazón, la cateterización cardiaca y la resonancia magnética ${ }^{2}$ que actualmente son de elección para identificar la magnitud de las anomalías presentes en el CS. En la actualidad este síndrome así como otras malformaciones cardiacas pueden diagnosticarse en la vida intrauterina a través de ecocardiografías prenatales. ${ }^{13}$ Así, entre 18 y 20 semanas de gestación es posible saber si el niño tiene o no algún problema en el corazón, específicamente en la válvula mitral, si éste se detecta antes de nacer o en los primeros meses de vida puede tratarse precozmente. Lo más complejo de este síndrome radica por lo regular en el compromiso de la válvula mitral; sin embargo, en muchos casos la coartación aórtica es reconocida antes de que se manifiesten otros defectos, llegando a enmascarar distintas anormalidades y en algunos pacientes el CS es diagnosticado por persistencia de los síntomas después de la reparación quirúrgica de la coartación. En cuanto al diagnóstico diferencial, los hallazgos del CS deben distinguirse del cor triatriatum izquierdo, debido a que su origen embriológico, morfología y complicaciones quirúrgicas son completamente diferentes.

Una vez establecido el diagnóstico el manejo quirúrgico es la corrección de las anomalías presentes. En las series de St. Louis, ${ }^{14}$ de Brown ${ }^{9}$ y Delmo ${ }^{15}$ en las que se conjunta un total de 100 pacientes con CS sometidos a cirugía, los resultados pueden ser favorables para la mayoría de ellos, siendo la severidad de la enfermedad mitral el factor más importante relacionado con la mortalidad, aunado a la necesidad de múltiples procedimientos operatorios. Los pacientes con CS tienen un pronóstico de vida acortado con mortalidad que alcanza $25 \%$ hasta hace pocos años. Los avances actuales en cirugía cardiotorácica han incrementado la supervivencia; no obstante, el pronóstico puede verse influido por complicaciones tales como: infección frecuente, ictus, insuficiencia cardiaca crónica, bloqueo cardiaco, taquiarritmias supraventriculares por dilatación de cavidades (no como en este caso, con síndrome de preexcitación tipo Wolff Parkinson White, asociación extraordinaria) y por la necesidad de varias cirugías a lo largo de la vida del paciente con la comorbilidad que ello implica.

\section{REFERENCIAS}

1. Shone JD, Sellers RD, Anderson RC, Adams P Jr, Lillehei CW, Edwards JE. The developmental complex of "parachute mitral valve", supravalvular ring of left atrium, subaortic stenosis, and coarctation of aorta. Am J Cardiol. 1963; 11: 714-725.

2. Roche KJ, Genieser NB, Ambrosino MM, Henry GL. MR findings in Shone's complex of left heart obstructive lesions. Pediatr Radiol. 1998; 28 (11): 841-845.

3. Brauner RA, Laks H, Drinkwater DC Jr, Scholl F, McCaffery S. Multiple left heart obstructions (Shone's anomaly) with mitral valve involvement: long-term surgical outcome. Ann Thorac Surg. 1997; 64 (3): 721-729.

4. Ikemba CM, Eidem BW, Fraley JK, Eapen RS, Pignatelli R, Ayres NA et al. Mitral valve morphology and morbidity/mortality in Shone's complex. Am J Cardiol. 2005; 95 (4): 541-543.

5. Ma XJ, Huang GY, Liang XC, Liu XQ, Jia B. Atypical Shone's complex diagnosed by echocardiography. Pediatr Cardiol. 2011; 32 (4): 442448.

6. Qamruddin S, Naqvi TZ. Advances in 3D echocardiography for mitral valve. Expert Rev Cardiovasc Ther. 2011; 9 (11): 1431-1443. 
7. Hakim FA, Kendall CB, Alharthi M, Mancina JC, Tajik JA, Mookadam F. Parachute mitral valve in adults-a systematic overview. Echocardiography. 2010; 27 (5): 581-586.

8. Rim Y, McPherson DD, Kim H. Effect of congenital anomalies of the papillary muscles on mitral valve function. J Med Biol Eng. 2015; 35 (1): 104-112.

9. Brown JW, Ruzmetov M, Vijay P, Hoyer MH, Girod D, Rodefeld MD et al. Operative results and outcomes in children with Shone's anomaly. Ann Thorac Surg. 2005; 79 (4): 1358-1365.

10. Narvencar KPS, Jaques e Costa AK, Patil VR. Shone's complex. JAPI. 2009; 57: 415-416.

11. Chandra S, Kharwar RB, Mundhekar A. Shone complex. J Cardiovasc Ultrasound. 2015; 23 (1): 54-55.
12. Purvis JA, Smyth S, Barr SH. Multi-modality imaging of an adult parachute mitral valve. J Am Soc Echocardiogr. 2011; 24 (3): 351.e1-3.

13. Zucker N, Levitas A, Zalzstein E. Prenatal diagnosis of Shone's syndrome: parental counseling and clinical outcome. Ultrasound Obstet Gynecol. 2004; 24 (6): 629-632.

14. St Louis JD, Bannan MM, Lutin WA, Wiles HB. Surgical strategies and outcomes in patients with Shone complex: a retrospective review. Ann Thorac Surg. 2007; 84 (4): 1357-1362; discussion 1362-1363.

15. Delmo-Walter EM, Van Praagh R, Miera O, Hetzer R. Repair of left ventricular inflow tract lesions in Shone's anomaly: valve growth and long-term outcome. Ann Thorac Surg. 2013; 95 (3): 948-955. 